0.28-0.61). The OGRS was a more respectable 0.69 (95\% CI $0.53-$ 0.85). In the HCR-20 high-risk group, AUC for VRAG was 0.67 (95\% CI 0.54-0.81) and OGRS 0.68 (95\% CI 0.64-0.81).

Perhaps there would be mileage in squeezing the fruit again in Buchanan's next study?

1 Cleckley H. The Mask of Sanity: An Attempt to Reinterpret the So-Called Psychopathic Personality. Mosby, 1941.

2 Hare RD. The Hare Psychopathy Checklist-Revised (2nd edn). Multi-Health Systems, 2003.

3 Seto M. Is more better? Combining actuarial risk scales to predict recidivism among adult sex offenders. Psychol Assess 2005; 17: 156-67.

4 Leon G. Assessing the validity and reliability of diagnostic and screening tests. In Epidemiology (3rd edn): 71-94. Elsevier Saunders, 2004.

5 Coid JW, Yang M, Ullrich S, Zhang T, Sizmur S, Farrington D, et al. Most items in structured risk assessment instruments do not predict violence. J Forens Psychiatry Psychol 2011; 22: 3-21.

6 Liu YY, Yang M, Ramsay M, Li XS, Coid JW. A comparison of logistic regression, classification and regression tree, and neural networks models in predicting violent re-offending. J Quant Criminol 2011; 27: 547-73.

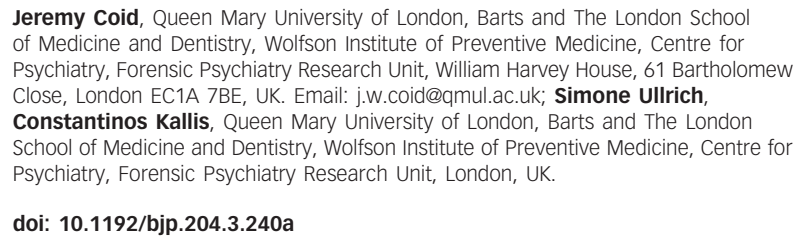

\section{Effectiveness of methadone treatment for heroin addiction}

Regarding Byford et al's paper, ${ }^{1}$ the authors present an analysis of the results of the Randomised Injectable Opiate Treatment Trial (RIOTT). ${ }^{2}$ Participants of RIOTT were very few in number - fewer than 45 individuals in each of the three arms of the study (injectable heroin, injectable methadone and 'optimised' oral methadone). It required 3 full years at 3 sites to screen 301 volunteers, of whom $127(40 \%)$ began the trial and only 89 completed the 26-week treatment protocol.

All of the participants had been receiving 'conventional' methadone treatment for more than 6 months and continued 'to inject "street" heroin regularly'. On average, they had had over four prior treatment episodes. Accordingly, it is reasonable to assume that the overriding motivation of those who volunteered was the hope of receiving injectable opiates, and it is likely that participant bias may have had a substantial impact on outcomes. Indeed, it is revealing that among those assigned to receive optimised oral methadone, $7(17 \%)$ never began the trial and of the remaining 35 only 24 were still enrolled 26 weeks later.

Some of the reported findings seem to underscore the severe limitations that must be kept in mind in drawing even the most tentative conclusions. For example, although the oral methadone group claimed to have committed roughly three times as many crimes as the intravenous methadone group (mean 21 v. 7 crimes), the latter group spent 15 times more nights in prison (mean 6.1 v. 0.4). Surely provision of oral methadone did not somehow make patients more successful in their criminal pursuits.

Perhaps inevitably, the limited ability to extrapolate has been ignored in the wider distribution of the findings. Thus, one report (which refers readers seeking more information to the Press Officer of King's College London, with which the principal author and five of the seven co-authors are affiliated) had the unqualified headline: 'Injectable opioid treatment for chronic heroin addiction more cost-effective than oral methadone', and claimed that 'total cost savings of providing injectable opiate treatment for this chronic group in England could be between $£ 29$ and $£ 59$ million per year. ${ }^{3}$

The criticisms noted above must not detract from the bottomline, common sense, conclusion with regard to injectable opioid treatment: in the interests of addicts as well as the general community, it is essential that those who respond poorly to treatment (any treatment) be provided information on and referral to the broadest possible array of alternative services.

1 Byford S, Barrett B, Metrebian N, Groshkova T, Cary M, Charles V, et al. Costeffectiveness of injectable opioid treatment $\mathrm{v}$. oral methadone for chronic heroin addiction. Br J Psychiatry 2013; 203: 341-9.

2 Strang J, Metrebian N, Lintzeris N, Potts L, Carnwath T, Mayet S, et al. Supervised injectable heroin or injectable methadone versus optimized oral methadone as treatment for chronic heroin addicts in England after persistent failure in orthodox treatment (RIOTT): a randomized trial. Lancet 2010; 375: 1885-95.

3 King's College London. Injectable opioid treatment for chronic heroin addiction more cost-effective than oral methadone. King's College London, 2013; 1 October (http://www.kcl.ac.uk/iop/news/records/2013/October/ Injectable-opioid-treatment-for-chronic-heroin-addiction-more-cost-effectivethan-oral-methadone.aspx).

Robert Newman, President Emeritus, Beth Israel Medical Center, New York, New York, USA. Email: rnewman@icaat.org

doi: 10.1192/bjp.204.3.241

Authors' reply: Newman rightly draws attention to the effectiveness of appropriately delivered methadone treatment for many people with heroin addiction worldwide over the past half-century. Our economic evaluation ${ }^{1}$ and the preceding report on the main findings from the RIOTT trial ${ }^{2}$ should not be considered an attack on the value of oral methadone to the majority who show substantial benefit from this treatment. ${ }^{3,4}$ Rather the RIOTT trial needs to be recognised for what it was an investigation of effectiveness and cost-effectiveness of alternative treatments in a subgroup of the treatment population with severe and chronic addiction who were not responding to oral methadone maintenance treatment.

It is also appropriate to inject a note of caution about the potential influence of expectations on trial participants. This limitation is inherent in any trial where the patient has a preference for which treatment arm they may be assigned to, and Newman is right that this has the potential to be a pronounced influence in the addiction treatment field. In fact, aware of this potential, we gathered some data from patients on their expectations and experiences of treatment within the trial, and this has recently been reported separately. ${ }^{5}$

Newman notes the modest sample size in this trial (total of 127 participants). This is a particular challenge in a field where treatment is intensive and expensive, and in countries which do not have a tradition of funding large treatment trials in the addictions field. We would nevertheless point out that the sample size was calculated in advance by the applicants for the original research award and was judged to be adequate to detect the expected effect size as defined in the protocol. $^{2}$

Newman highlights a further limitation of sample size in this highly variable population, using the example of criminal activity. Although the oral methadone group reported committing a much higher number of crimes than the injectable methadone group, the latter group spent more nights in prison. However, the total number of participants spending any time in prison $(n=6 ; 5 \%)$ is extremely small relative to the number reporting any criminal activity $(n=50 ; 42 \%)$, so it would be inappropriate to try and come to any comparative conclusions. 
In conclusion, we acknowledge the limitations of research in this complex subgroup with chronic heroin addiction and also the evidence of benefit from oral methadone in the broader population of people addicted to the drug. However, we consider the important findings reported in the paper are that, for this subgroup doing persistently badly on oral methadone treatment, it is important for clinicians to work with their patients to explore alternative options, such as injectable treatments, which may achieve health benefits not being achieved in the expected manner with the orthodox first-line treatment, and which may achieve this health benefit in a more cost-effective manner. Such personalisation of treatment plans is important but is currently being hindered by the cost implications of providing injectable alternatives and a previous lack of evidence of cost-effectiveness.

1 Byford S, Barrett B, Metrebian N, Groshkova T, Cary M, Charles V, et al. Cost-effectiveness of injectable opioid treatment $v$. oral methadone for chronic heroin addiction. Br J Psychiatry 2013; 203: 341-9.

2 Strang J, Metrebian N, Lintzeris N, Potts L, Carnwath T, Mayet S, et al. Supervised injectable heroin or injectable methadone versus optimized oral methadone as treatment for chronic heroin addicts in England after persistent failure in orthodox treatment (RIOTT): a randomized trial. Lancet 2010; 375: 1885-95.

3 National Institute for Health and Clinical Excellence. Methadone and Buprenorphine for the Management of Opioid Dependence (NICE Technology Appraisal TA114). NICE, 2007

4 Strang J, Babor T, Caulkins J, Fischer B, Foxcroft D, Humphreys K. Drug policy and the public good: evidence for effective interventions. Lancet 2012; 379 71-83.

5 Groshkova T, Metrebian N, Hallam C, Charles V, Martin A, Forzisi L, et al. Treatment expectations and satisfaction of treatment-refractory opioiddependent patients in RIOTT, the Randomised Injectable Opiate Treatment Trial, the UK's first supervised injectable maintenance clinics. Drug Alcohol Rev 2013; 32: 566-73.

Sarah Byford, Centre for the Economics of Mental and Physical Health, Box P024 Institute of Psychiatry, De Crespigny Park, London SE5 8AF, UK. Email: s.byford@kcl.ac.uk; Barbara Barrett, Centre for the Economics of Mental and Physical Health, Nicola Metrebian, Addictions Department, National Addiction Centre, Institute of Psychiatry, King's College London, UK; Teodora Groshkova, Centre, Institute of Psychiatry, King's College London, UK; Teodora Groshkova,
European Monitoring Centre for Drugs and Drug Addiction, Lisbon, Portugal; Maria European Monitoring Centre for Drugs and Drug Addiction, Lisbon, Portugal; Maria
Cary, Centre for the Economics of Mental and Physical Health, Vikki Charles, Addictions Department, National Addiction Centre, Institute of Psychiatry, King's College London, UK; Nicholas Lintzeris, The Langton Centre, South Eastern Sydney Local Health District, NSW Health, Australia; John Strang, Addictions Department, National Addiction Centre, Institute of Psychiatry, King's College London, UK

doi: 10.1192/bjp.204.3.241a

\section{Psychosis as a failure of reality testing}

Garety \& Freeman present a timely review on the nature of delusional experience. ${ }^{1}$ Their conclusion regarding the need to focus on individual features of psychosis seems apt. The presented overview of cognitive and affective mechanisms influencing delusion development seems, however, to overlook an essential component of delusional experience; that psychotic symptoms, including delusions, at their heart represent a failure of reality testing.

The description of jumping to conclusions, together with the probabilistic reasoning task methodology, appear to rely on a logical chain of thought progression and conclusion - what Campbell has referred to as an empiricist understanding. ${ }^{2}$ This approach, however, does not take into account the nature of conclusions reached in delusional belief. Conclusions reached on seeing two, or fewer, coloured counters seem quite distinct from classic examples of delusional perception: 'I saw the traffic lights turn green and realised that the world would end'. Campbell's alternative rationalist approach presents the person with delusions as having experienced a complete rearrangement of their framework propositions, or underlying background world beliefs.
Such a fundamental shift in a world-view model can go some way to explaining the fantastical nature of conclusions reached, or the failure of reality testing present in psychosis.

Campbell's arguments have not gone unchallenged. ${ }^{3}$ However, what they do highlight is a need for careful consideration as to the manner in which delusional beliefs are formed. Garety \& Freeman describe the psychoanalytic thinking in relation to defence mechanisms in the development of persecutory delusional belief. Psychotic defence concepts, wherein the individual denies or distorts reality to defend against trauma, provide one possible lens through which psychotic experiences can be viewed. ${ }^{4,5}$

Garety \& Freeman's conclusion relating to the infancy of research into the nature of delusion, and its having been overshadowed by focus on the larger concept of schizophrenia, highlights the need for further research. Future research will need to provide some account for the distortion of reality that seems central to the experience of psychosis.

1 Garety PA, Freeman D. The past and future of delusions research: from the inexplicable to the treatable. Br J Psychiatry 2013; 203: 327-33.

2 Campbell J. Rationality, meaning, and the analysis of delusion. Philos Psychiatr Psychol 2001; 8: 89-100.

3 Bayne T, Pacherie E. Bottom-up or top-down: Campbell's rationalist account of monothematic delusions. Philos Psychiatr Psychol 2004; 11: 1-11.

4 Hingley SM. Psychodynamic perspectives on psychosis and psychotherapy. I: Theory. Br J Med Psychol 1997; 70: 301-12.

5 Martindale B, Summers A. The psychodynamics of psychosis. Adv Psychiatr Treat 2013; 19: 124-31.

Andrew Shepherd, Doctor, University of Manchester, UK Email: andrew.shepherd3@nhs.net

doi: 10.1192/bjp.204.3.242

Authors' reply: We differ in our approach from that taken by Shepherd, in that we are advocating an empirical approach which posits hypotheses that can be and are tested. Our review of over 200 studies demonstrates how much has been learned by testing hypotheses, amassing evidence and replicating findings. ${ }^{1}$ Thus there is now strong and consistent evidence that delusions are associated with biases in reasoning, such as are assessed by experimental tasks and reliable interviews. These findings are important and provide an explanation of the failure to take on board all the evidence - or a failure of reality testing, as Shepherd puts it. We now therefore have secure knowledge of specific reasoning processes which may be targeted in treatment. ${ }^{2}$

We do not agree that world beliefs are fundamentally rearranged in people with delusions. Rather, the person's delusions can be shown to build on the pre-existing thoughts about self and world, and are actually typically preceded by periods of anxious worry. ${ }^{3}$ Traditional views of sudden dramatic changes are not in general supported by the evidence. Although we show that there is clear evidence of the importance of emotional processes - and in some cases this can be linked to childhood trauma - we do not conclude that the delusion represents a defence. The psychoanalytic defence accounts are not supported by the evidence. Rather, anxiety and depression - and negative views of self and others - are risk factors for and commonly expressed by patients with delusions. ${ }^{4}$ We consider that these research findings render delusions explicable, and may have implications for the way all clinicians engage with people with delusions.

We advocate that there is now enough certainty in the evidence base for concerted efforts to translate them into targeted treatments for delusions. It is through further trials, drawing on the evidence base which identifies mechanisms underpinning 